\title{
Application of Digital Image Correlation and Geodetic Displacement Measuring Methods to Monitor Water Dam Behavior under Dynamic Load
}

\author{
Janina Zaczek-Peplinska ${ }^{1}$, Maria Elżbieta Kowalska ${ }^{1}$, Krzysztof Malowany $^{2}$ and Marcin Malesa ${ }^{2}$ \\ 1. Faculty of Geodesy and Cartography, Warsaw University of Technology, Warszawa 00-661, Poland \\ 2. Institute of Micromechanics and Photonics, Warsaw University of Technology, Warszawa 00-661, Poland
}

\begin{abstract}
This paper presents experiment results of the measurement conducted at the Rożnów Dam power plant. For a course of starting and operating of turbo-plants, downstream face of the dam was monitored in relation to its eventual displacements on direction parallel to the construction axis. For the purpose of the experiment, geodetic measurement techniques and 2D DIC (digital image correlation) method (utilizing photographs of the object recorded with digital camera) were compared with regard to credibility, efficiency and accuracy. The vertical and horizontal displacements were monitored by tachometers measurements. The deformations in $x$-axis and $y$-axis on the wall surface was monitored by 2D DIC. It has been noticed that 2D DIC method is a surface method, continuous - not discreet. It allows for continuous observations of surface deformations, which is not possible in case of tachemetric measurements. Despite many advantages, the 2D DIC method lacks unambiguous evaluation of precision and relevance of designated displacements, which is rather significant for possibilities of utilization in technical control of large engineered objects. It should be also marked that the tachometric method is more reliable but is more laborious. Research of this type might comprise additional element for the assessment of the influence of dynamic loads, such as activating turbine water flow, onto the overall condition of the surveyed structure.
\end{abstract}

Key words: Hydrotechnical structure, structure dynamic load, engineering object displacement, geodetic displacement measurements, digital image correlation.

\section{Introduction}

Typical hydrotechnical structures are large sized objects of considerable mass. Their specific characteristic is constant contact with water, especially surface water, whilst its main function of impounding water causes constant long-term load of water onto structure and its base, which becomes the most significant influence onto object. Additional factor loading dam structure is operation of power plant - enabling a flow of water through wide section technological pipes and starting-up a turbo-plant (a combined unit of hydraulic turbines and electrical generators weighting several tones) create elastic

Corresponding author: Janina Zaczek-Peplinska, Ph.D., research fields: geodesy and cartography and engineering geodesy. E-mail: j.peplinska@gik.pw.edu.pl. deformations of dam power plant sections and power-plant base (Fig. 1). Effectively, under influence of appearing and disappearing dynamic load, neighbouring dam sections yield to displacements, absorbed by expansion joints. Periodicity of electricity production resulting from daily changes in electricity demand causes multiple loading changes, followed by cyclical displacements of structural elements.

During the course of periodical monitoring survey of concrete dam in Rożnów (Poland) in August 2013 described earlier, phenomenon of elastic deformations of adjacent to the power plant dam sections was observed. Slight movements of sections were noticed in sequences of photographs taken during observations of the downstream face of the dam. Images were processed using DIC (digital correlation method), 


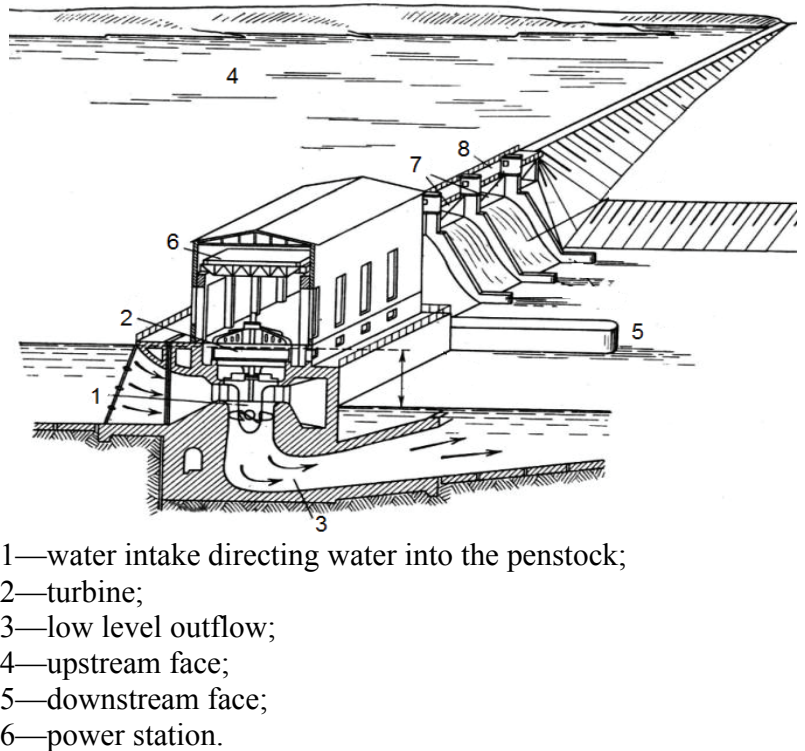

Fig. 1 Cross section of hydroelectric power plant water dam section [1].

obtaining longitudinal displacement graphs (in direction parallel to the dam crown and perpendicular to the average plane of water flowing through piping leading to the turbine). Fig. 2 presented direction of observed changes of displacements. Results were confirmed by conducted at the same time geodetic tachometric measurements, by defining vertical and horizontal displacements of control points based on changes of their coordinates within objects coordinate system.

The both techniques were selected because each of them gives different results. The classical geodetic methods allow to determine displacements of characteristic points of the structure and the 2D DIC method shows deformations in certain parts of the surface. Different methods and different results can to be used to assessment the same parameters of work of the objects concrete sections.

As it was already mentioned, starting a power plant generates dynamic load, resulting in a variable in time movement of adjacent sections and clear return to the initial state, after balancing loads.

The main goal of analysis presented in this paper was confirmation of occurrence of the phenomenon of the dam sections displacement during operation of the power plant, as well as comparison of two measuring methods utilized in different disciplines. Geodetic survey commonly used for technical monitoring of water dams $[2,3]$ was adopted as a reference.

\section{Concrete Dam in Rożnów}

Subject of conducted research is a heavy concrete dam located on $80,000 \mathrm{~km}$ of Dunajec River in Rożnów village (Fig. 3).

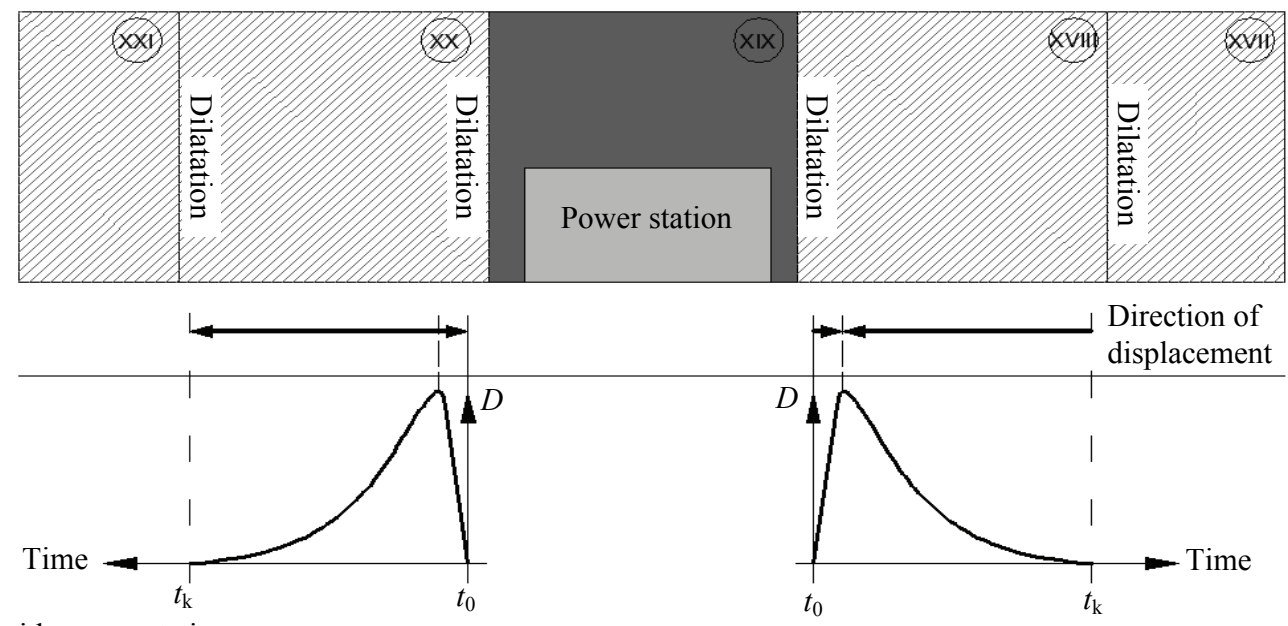

XIX - section with power station;

XVIII, XX - sections adjacent to the power station, liable to displacements;

XVII, XXI-successive sections;

$t_{0}$ - starting-up moment of the power plant operation (release of water);

$t_{x}-$ moment of the movement termination;

$D$-value of the observed movement.

Fig. 2 Diagram of observed structural displacements during power-plant operation. 


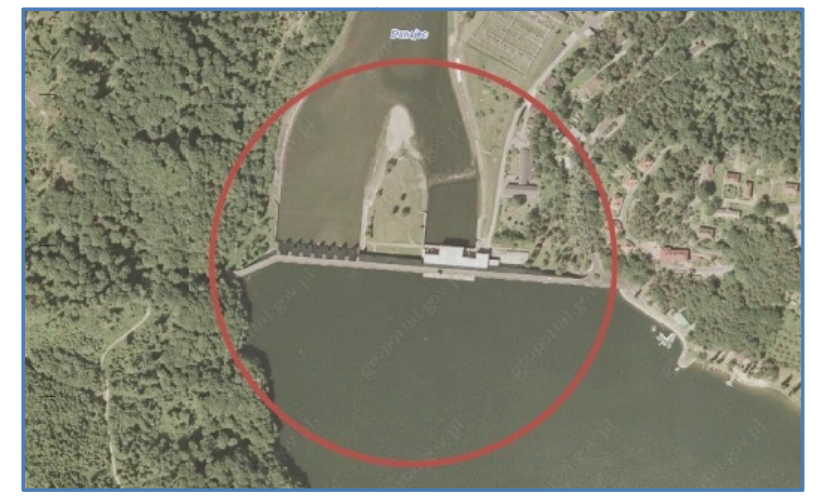

Fig. 3 Rożnów Dam.

Source: Geoportal.

Rożnów Dam is a first Class hydrotechnical object (Ordinance of Polish Ministry of Environment of April 20, 2007 regarding technical condition requirements to be fulfilled by hydrotechnical buildings and their locations). Rożnów Dam is built of 44 sections:

- 20 non-overflow dam sections, each 15-m wide. Non-overflow Sections XVII and XVIII are shown in Fig. 4;

- 12 non-overflow sections located within left abutment, 5-m and 7.5-m wide;

- seven overflow sections, five of which were fitted with bottom outlets;

- five electric power plant sections, fitted with Kaplan water-turbines (four sections) and engine room (one section). Plant Sections XIX and XX are shown in Fig. 4. Foundation foot for each sections was constructed with 1:10 gradient, declined towards the high water. Sealing of the ground base was executed in form of three rows of cement solution injections (cement slurry).

\section{Methodology}

\subsection{Displacements Measurements with 2D Digital} Image Correlation

\subsubsection{D Digital Image Correlation}

DIC (digital image correlation) is a non-coherent light-based method which enables full-field measurements of displacements and determination of strains of different objects [3]. Regular white-light images of a test object undergoing some sort of deformation or displacement are recorded in a sequence. The analysis of captured images are performed automatically by the software, which divides the region of interest in a reference image into discrete blocks (subsets). Those are subsequently matched against similar subsets in an another image from the sequence, using a correlation criterion [3] (Fig. 5). Sutton et al. [4] proposed the SSD (sum of square difference) of intensity values as a basic correlation criterion, however, the most advanced and the most computationally demanding is the ZNSSD (zero-mean normalised sum of square difference) criterion:

$$
\begin{aligned}
& x_{\mathrm{ZNSSD}}^{2}=\Sigma_{i}\left(\left(\frac{\sum_{i} \bar{G}\left(p_{i}\right) \bar{F}\left(p_{i}\right)}{\Sigma_{i} \bar{G}\left(p_{i}\right)^{2}} G\left(p_{i}\right)-\right.\right. \\
& \left.\left.\bar{G} \frac{\Sigma_{i} \bar{G}\left(p_{i}\right) \bar{F}\left(p_{i}\right)}{\sum_{i} \bar{G}\left(p_{i}\right)^{2}}\right)-F\left(p_{i}\right)+\bar{F}\right)^{2}
\end{aligned}
$$

The difference in position between a reference subset and its matched object equivalent defines the displacement vector at the subset's origin. Repeating this process over the area of interest yields a displacement map that is sampled as defined by the

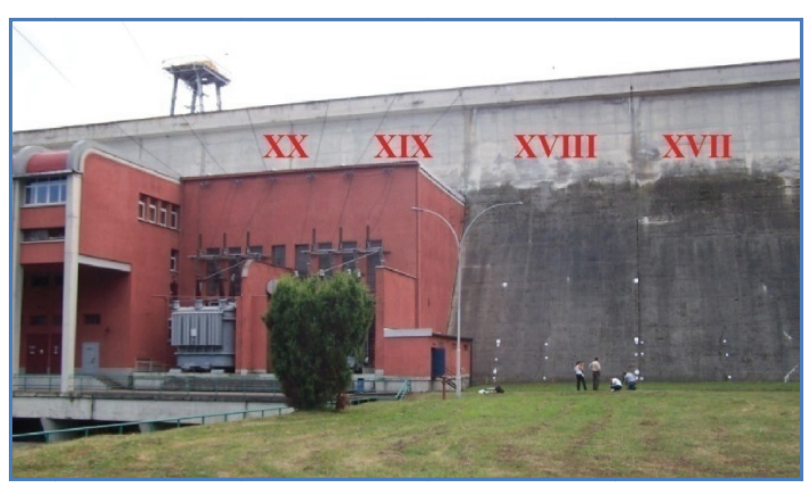

Fig. 4 View of downstream face onto Sections XVII-XX. Source: author's archive.

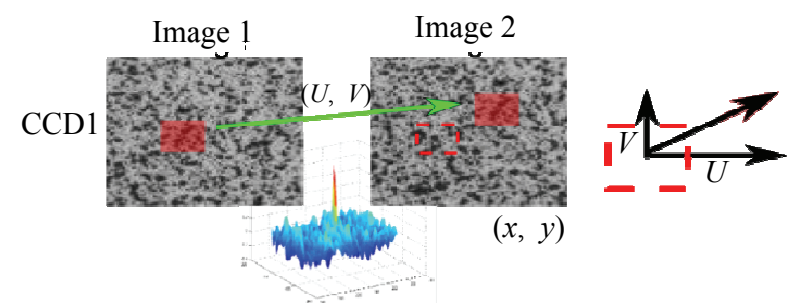

Fig. 5 Subset matching algorithm in 2D DIC. 
subset size another separation of subsequent subsets selected for analysis (step/size). The most distinctive characteristic of DIC is that the object surface needs to exhibit a random intensity distribution (called "speckle pattern") - that way, each subset is sure to be unique and can be unambiguously identified.

\subsubsection{Measurement Setup}

Measurements were performed with the 2D DIC system that comprises AVT Pike F-1600 $(4,872 \times 3,248$ pixels $)$ cameras equipped with $50-\mathrm{mm}$ lenses, observing AOI (area of interest), at the size of 15-m wide and 9-m high (Fig. 6a), on the object Section XVIII (Figs. 4 and 6). In experimental mechanics for measurements performed with DIC techniques, surface of measured object is modified with the paint to obtain speckle pattern. During the measurement of dam, the degradation of dam concrete surface (e.g., discoloration, small cracks and detachment, Figs. 6b-6d) formed natural speckle pattern.

\subsubsection{Transformation of the Coordinate System}

The measured object was observed from the ground, and thus it was impossible to ensure perpendicularity between the object and the optical axis of the camera. The angle between the optical axis and the measured object would distort the obtained displacement maps significantly by the perspective error. In order to avoid this error, all images of the sequence have been transformed so that the object is observed by the camera perfectly perpendicular to the object (image rectification procedure [5]). This has been done by applying homography transform to all images. The homography (also called "projective transformation") $[5,6]$ is a geometrical transformation between two sets of coplanar points. The homography maps straight lines into straight lines and preserves an incidence and a cross-ratio. It does not preserve sizes nor angles of objects. The homography can be used to describe a perspective projection when a point of view of the observer changes [7]. The homography can be represented by $3 \times 3$ homography matrix. The matrix can be calculated from a corresponds of at least four points viewed in two images.

In the presented measurements, the homography matrix has been used to rectify the images of the measured object. Positions of markers in real-world coordinates were known from surveying. In the first step, the markers applied to the measured object have been detected in all images of the series. Next, virtual positions of all markers have been generated. The virtual positions have been generated so to represent corners of the rectangle. From point to point correspondence between the markers and virtual positions, the homography matrix has been calculated. This was done with utilization of the DLT (direct linear transformation) method enclosed in RANSAC algorithm [8]. Finally, the calculated matrix has been applied to all images of the series (Fig. 7).

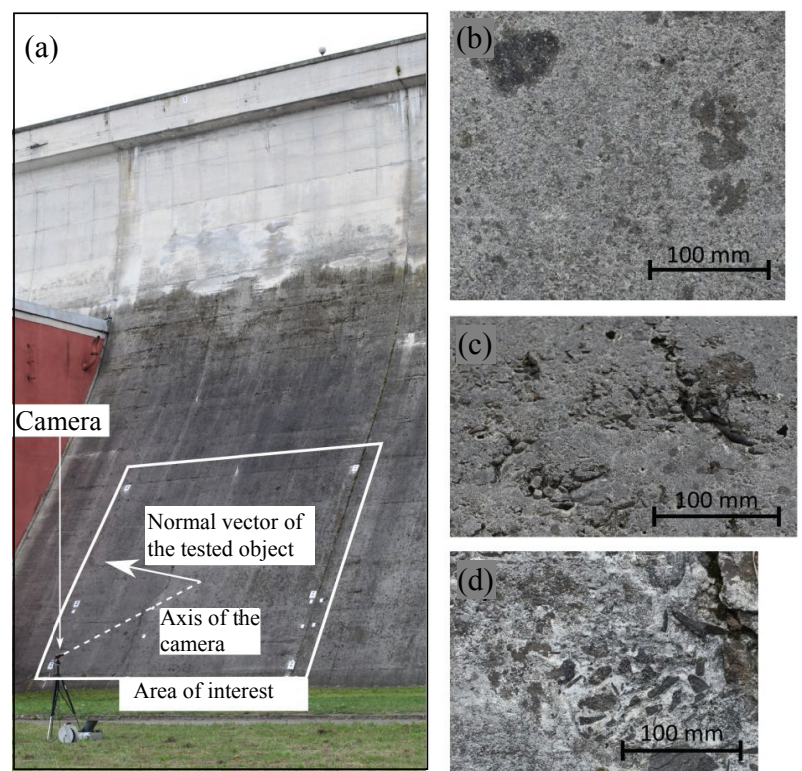

Fig. 6 2D DIC set up with the measured object: (a) natural surface degradation of dam; (b) discoloration; (c) small cracks; (d) detachment.

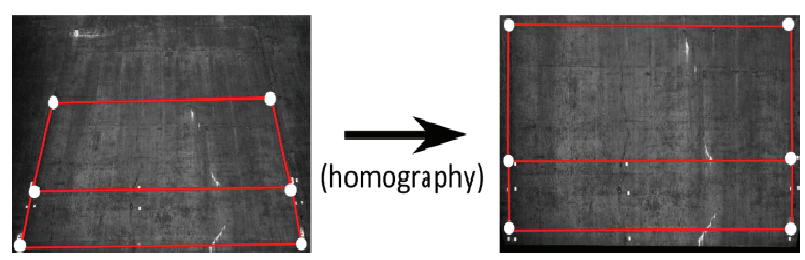

Fig. 7 Image of the measured object before and after homography transformation. Locations of markers applied to the object's surface are indicated in both images. 


\subsubsection{Digital Image Correlation 2D Results}

From captured and transformed images, the displacement maps in two directions were calculated. Displacements in the $x$-direction are given as $u$ (in pixels) and after scaling are expressed as $U$ (in $\mathrm{mm}$ ). Similarly, displacements in the $y$-direction are given as $v$ (in pixels) and $V$ (in mm). In Fig. 8, the displacement map $U$ at maximum value is shown, with the orientation of coordinate system and marked points (P1-P4). Data obtained from marked points were used for quantitative analysis (Fig. 9).

\subsection{Measurements of Dam Displacements Using Geodetic Method}

In order to research the behavior of the adjacent to the power plant XVIII dam section and confirmation of results obtained with DIC technique, three series of tacheometric measurement were conducted. Consecutive measurements were performed using following pattern: (1) $30 \mathrm{~min}$ before starting up turbines; (2) immediately after starting up water flow; (3) 30 min after starting up. Performing measurements in 30-min intervals allowed to determine dislocations of points indicated on the concrete surface. Additionally, analysis of obtained results allowed for

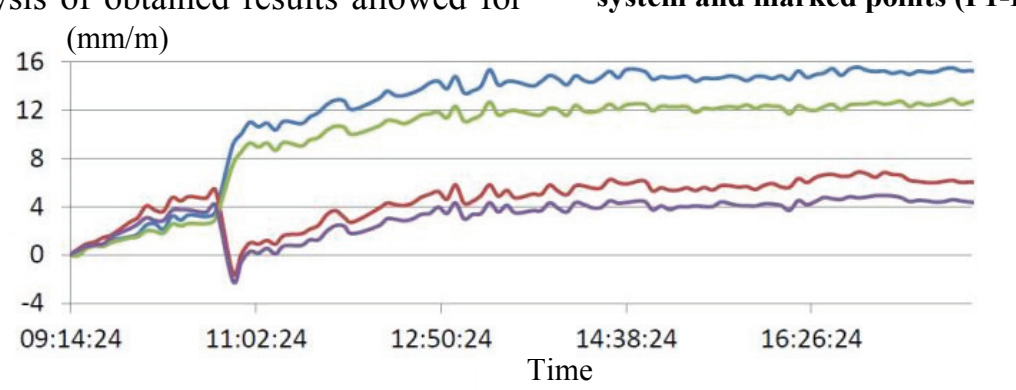

$$
\begin{aligned}
& -U(\mathrm{P} 1) \\
& -U(\mathrm{P} 2) \\
& -U(\mathrm{P} 3) \\
& -U(\mathrm{P} 4)
\end{aligned}
$$

(a)

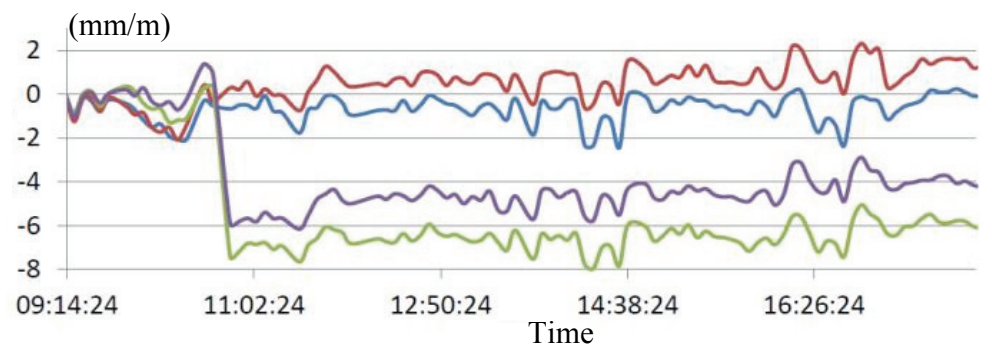

$$
\begin{aligned}
& -U(\mathrm{P} 1) \\
& -U(\mathrm{P} 2) \\
& -U(\mathrm{P} 3) \\
& -U(\mathrm{P} 4)
\end{aligned}
$$

(b)

Fig. 9 Observed with 2D DIC method's deformations $U \& V$ of marked points P1-P4: (a) deformations along the $x$-axis; (b) deformations along the $y$-axis. 


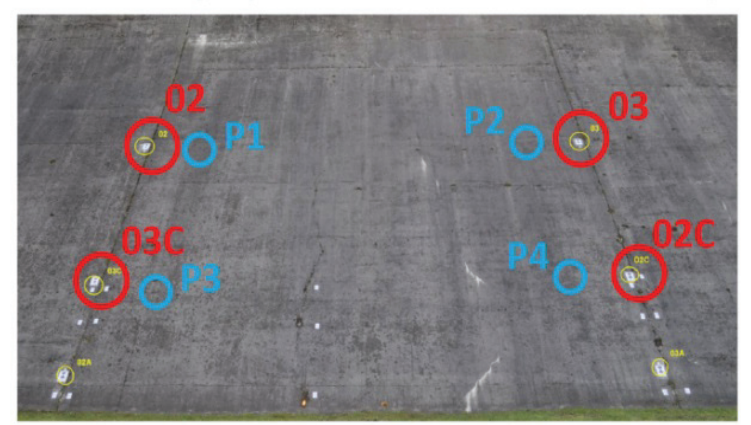

Points displacement determined using geodetic method Points displacement determined using DIC method

Fig. 10 Control points arrangement: displacements of $\mathrm{O2}$, O2C, O3, O3C points determined with geodetic method, deformations of P1-P4 points established using 2D DIC method.

arranged at the same levels. For the research, four points located in the middle part of the section were used (Fig. 10).

Angle and distance observations were performed using Leica1202+ instrument at 1-s accuracy, from single position related to four stabile reference points located around $100 \mathrm{~m}$ away from the dam. Measurements results were elaborated using coordinate difference method [9]. Obtained results of point displacements are presented on Figs. 11-12, which present accordingly values of determined horizontal displacements and values of determined vertical displacements of control points. A movement of XVIII and XVII dam sections, directed away from the power station, can be observed on graphs. Authors call movement of such character as "displacement parallel to dam crown axis directed away from the power station". Defined horizontal displacements, especially element perpendicular to the objects crown axis, indicate subtle periodical inclination of the dam section in the direction of high water. This confirms values of vertical displacements. Points located higher (O2 and $\mathrm{O} 3$ ) have been lowered (about $1 \mathrm{~mm}$ ), whilst points in the lower part of the wall (O2C and $\mathrm{O} 3 \mathrm{C}$ ) have been raised (about $2 \mathrm{~mm}$ ). All observed displacements are eventually expired. Returning to the state before the start-up to the power plant occurred within $1 \mathrm{~h}$ from the beginning of plant operation. During this time, measurements of control points located on the rest of the sections were performed, and ambient temperature was measured. Displacements of
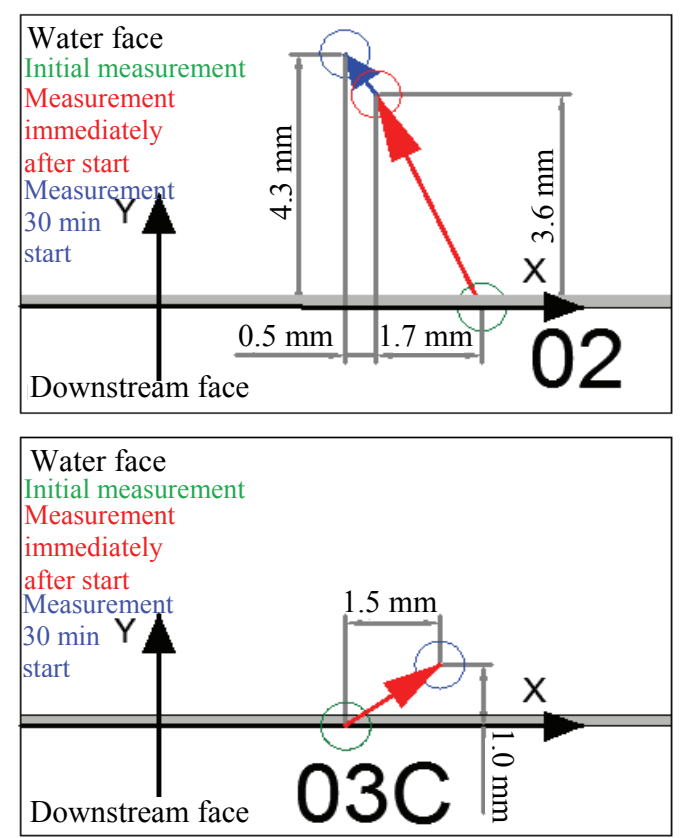

Fig. 11 Observed horizontal displacement values.

Green - initial state, 30 min before power plant's start-up; Yellow-state during starting up turbo-plants; Blue - state 30 min after starting up.

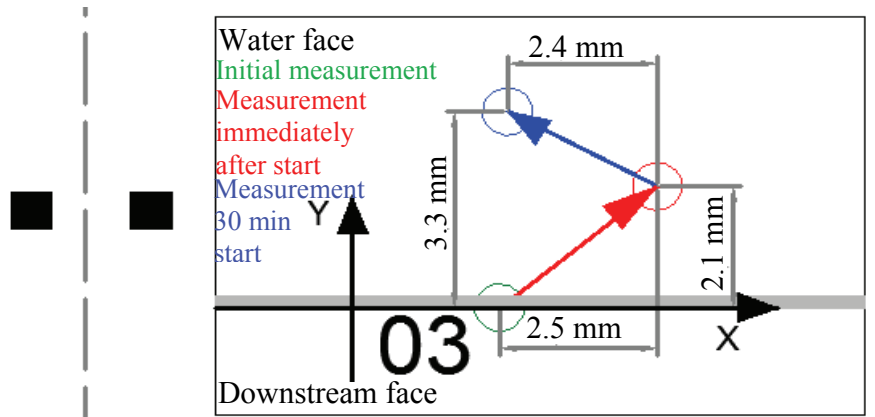

Downstream face

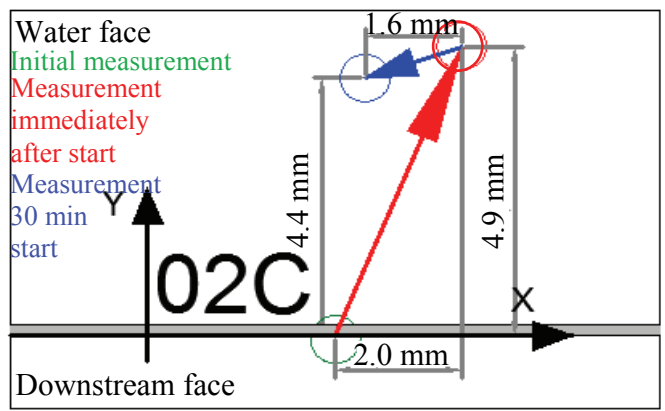



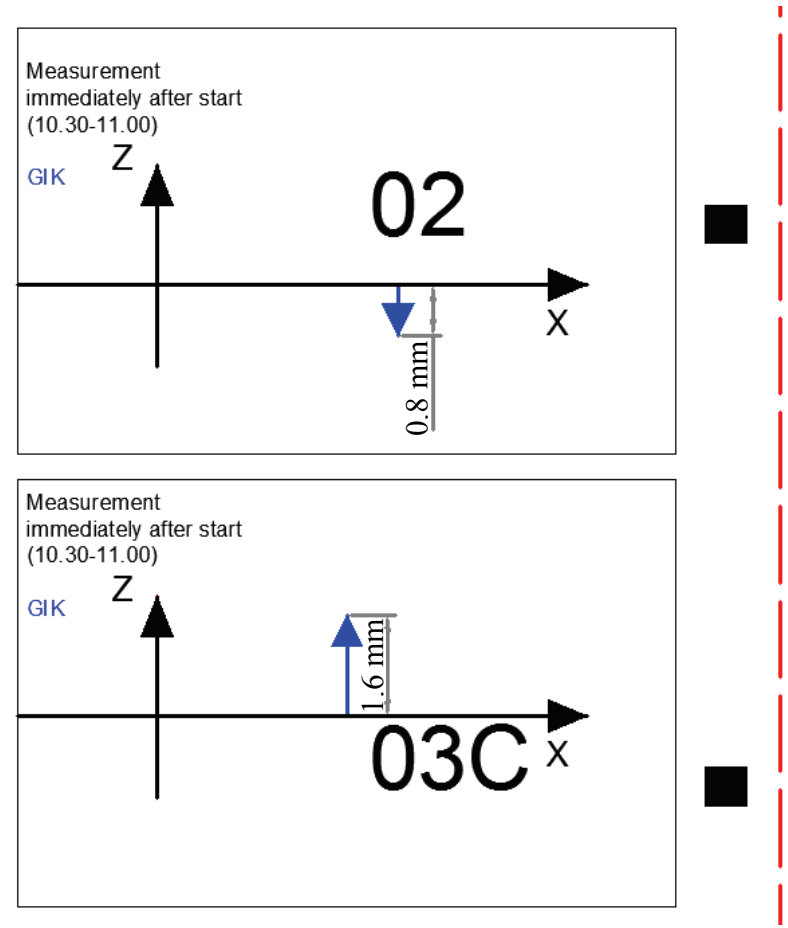

Fig. 12 Section XVIII-XVII: Observed vertical displacement values in a period of time between 30 min before starting and moment of starting.

Power Station - Section XIX $\quad$ Section XVIII | XVII

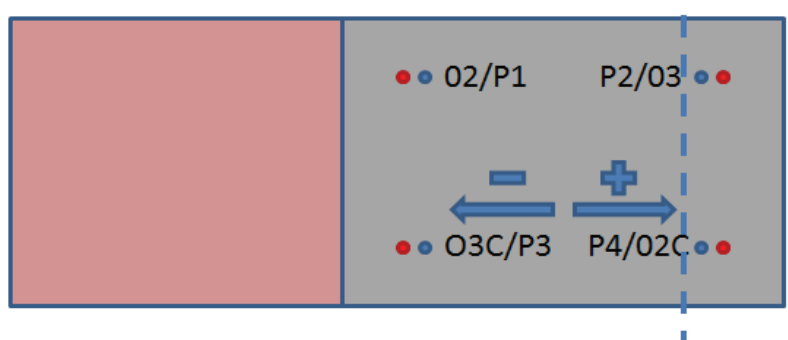

Fig. 13 Diagram of uniform definition of horizontal displacement direction (along the $x$-axis).

periphery sections were not affirmed, while temperature of concrete surface was constantly around $17 \sim 18{ }^{\circ} \mathrm{C}$. Through the presence of expansion gap within the whole structure, it can be suspected that interaction caused by operating turbo-plants was transferred to the sections structure by the objects foundation or sub-base.

\section{Comparison of Results Obtained Using Both Measurement Methods}

Obtained results, based on measurements performed with both methods, geodetic (based on registration/values of observed direction and distance

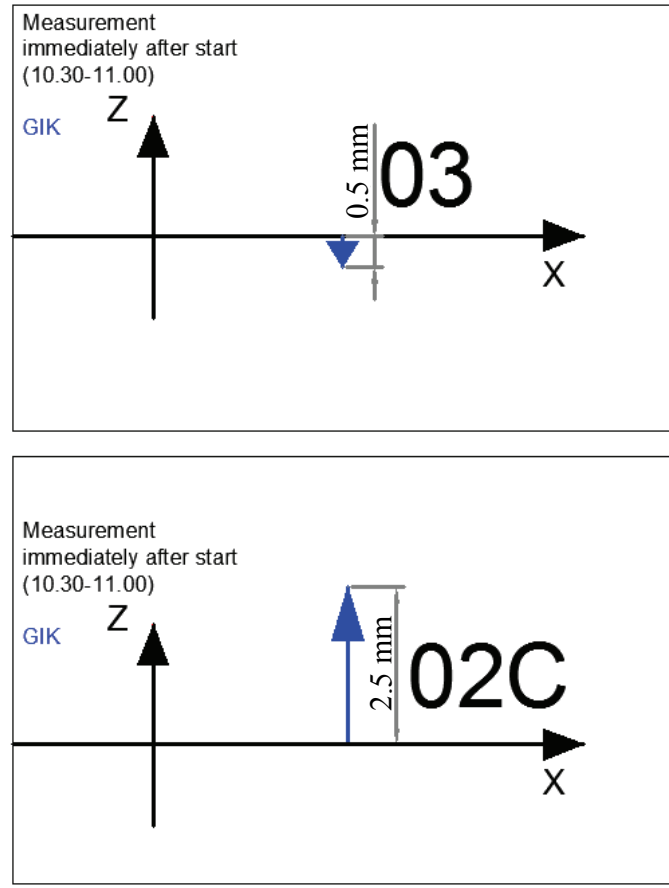

change) and DIC 2D characterize with high conformity. It is important to emphasize that 2D DIC method provides information about deformations in image plane only (in two directions: $x, y$ ), whereas tacheometric method gives displacements in three directions $(x, y, z)$. In order to compare the results of both methods, horizontal displacement values in direction parallel to the dam crown axis (along the $x$-axis) and vertical displacement values were taken into consideration.

\subsection{Longitudinal Displacements}

In order to compare established values of longitudinal displacements, in this work, a certain rule was adopted so that displacements towards the power station obtain negative sign, whereas away from the power station obtain a positive sign (Fig. 13). On a base of measurements with both methods, displacements of eight different points were determined (four for each method) and arranged in pairs located close together (Fig. 13), hence Figs. 14 and 15 show slight differences in conformity for measured values. For each of the 

Methods to Monitor Water Dam Behavior under Dynamic Load
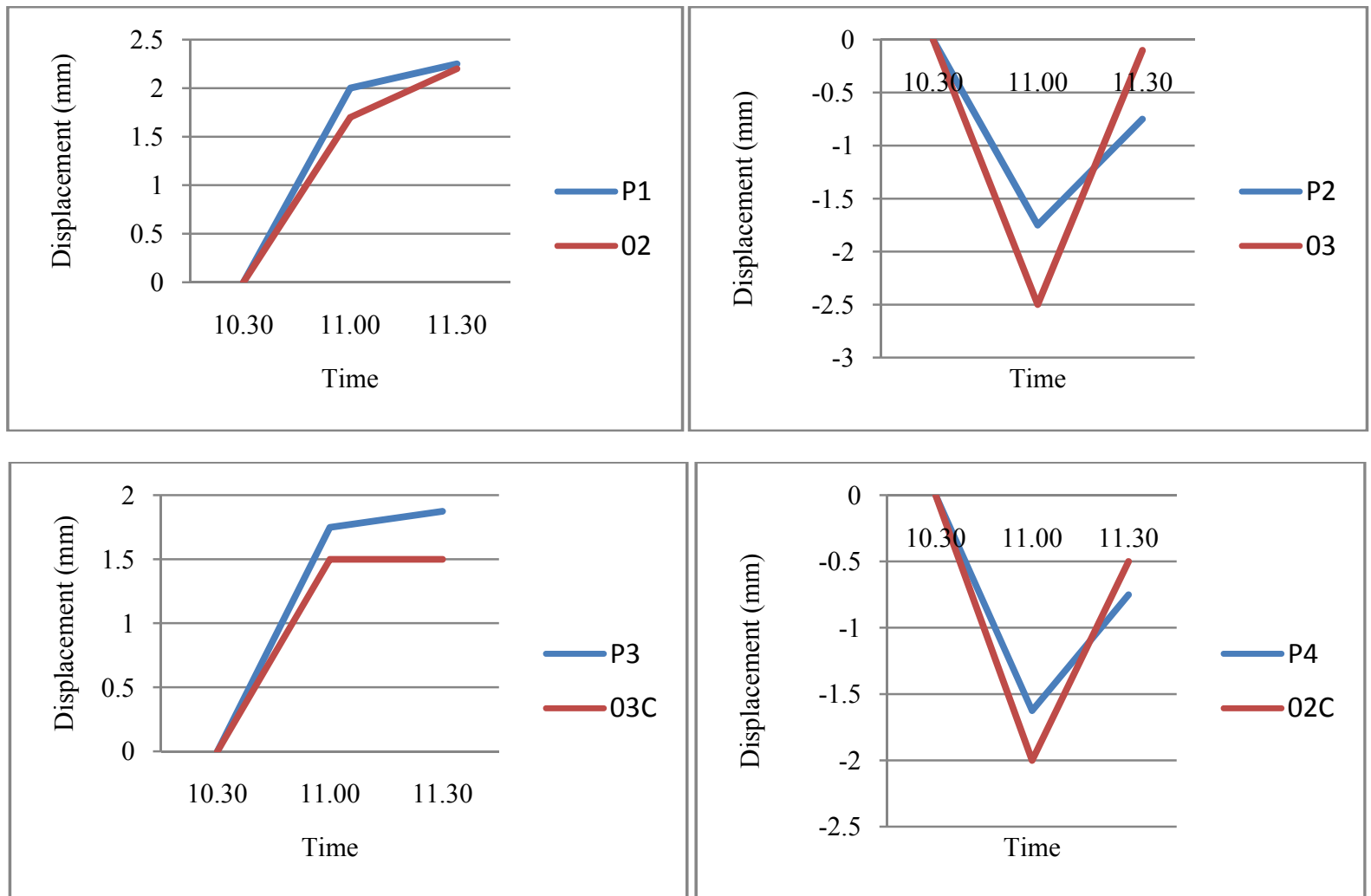

Fig. 14 Values of longitudinal displacements (in mm) obtained using geodetic measurements method (red color) and DIC method (blue color).
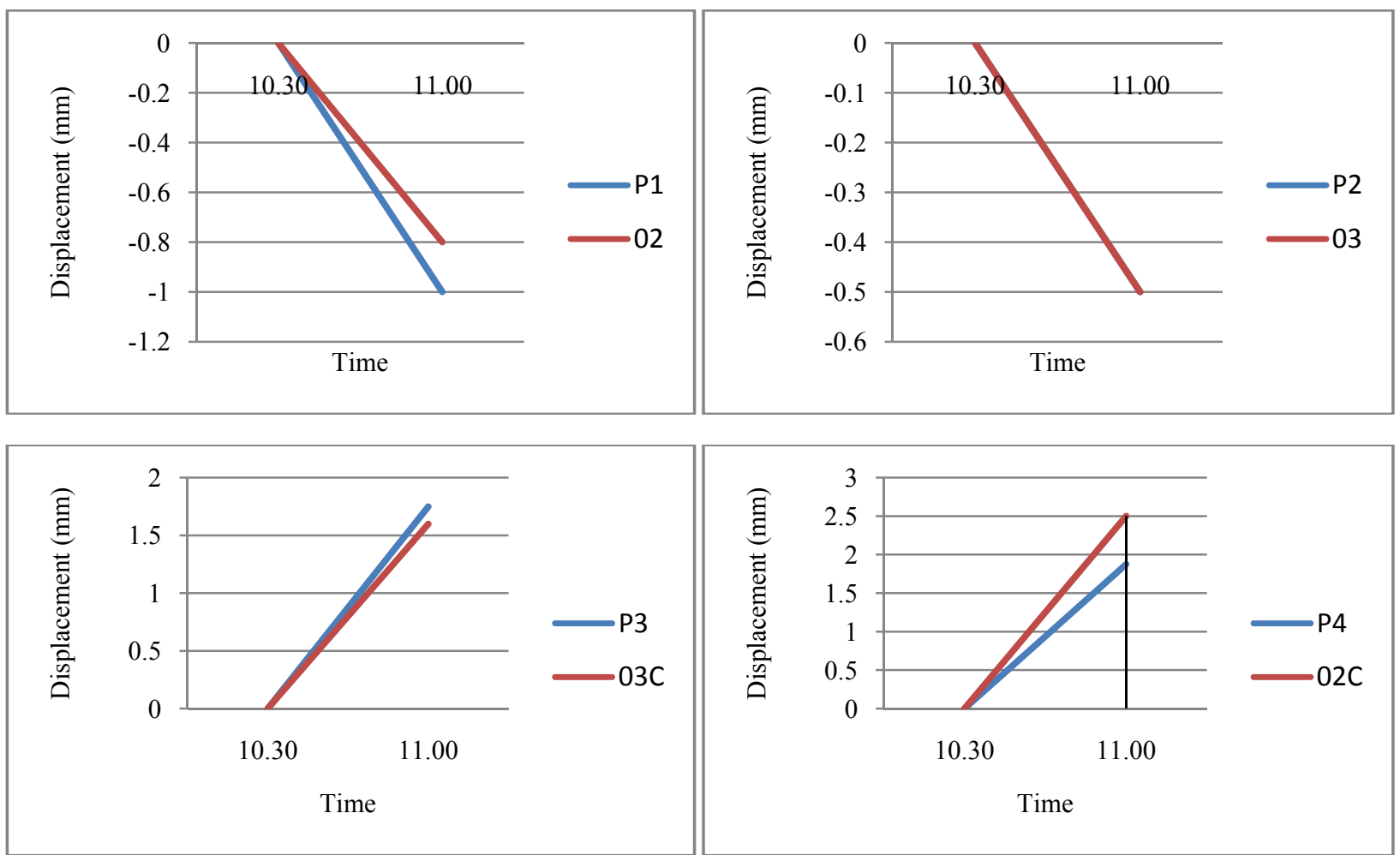

Fig. 15 Values of vertical displacements (in mm) obtained using geodetic measurements method (red color) and DIC method (blue color). 
Table 1 Comparison of vertical displacement values determined with two comparable methods.

\begin{tabular}{lll}
\hline Control Points & Vertical displacements from 2D DIC method $(\mathrm{mm})$ & Vertical displacements from tacheometry $(\mathrm{mm})$ \\
& $10.30 \sim 11.00$ & $10.30 \sim 11.00$ \\
\hline P1/02 & -1.0 & -0.8 \\
P2/03 & -0.5 & -0.5 \\
P3/03C & 1.8 & 1.6 \\
P4/02C & 1.9 & 2.5 \\
\hline
\end{tabular}

Table 2 Appraisal of compared measurement methods.

\begin{tabular}{lll}
\hline Description & Tacheometry (geodetic method) & 2D DIC method \\
\hline Determination of horizontal displacements (mm) & $\checkmark$ & $\times$ \\
Determination of longitudinal displacements (parallel to the objects & $\checkmark$ & $\checkmark$ \\
crown along $x$-axis) & $\checkmark$ & $\checkmark$ \\
Determination of vertical displacements & $\checkmark$ & $\times$ \\
Determination of 3D displacements (mm) & $\times$ & $\checkmark$ \\
Determination of 2D deformations (in vertical plane) (mm/m) & $\times$ & $\checkmark$ \\
Continuous visualization of surface deformations & $\times$ & $\times / \checkmark$ \\
Evaluation of accuracy and relevance of determined displacements & $\checkmark$ & $\checkmark$ \\
Stability of survey position & $\checkmark$ & $\checkmark$ \\
Labor-intensity & $\times$ & $\checkmark$ \\
Effectiveness & $\checkmark$ & \\
\hline
\end{tabular}

" $\mathbf{x}$ " stands for negative;

" $\checkmark "$ stands for positive score.

graphs, blue color represents displacements registered with 2D DIC method, and red color indicates displacements determined with tacheometric method. Results obtained with both methods characterize high conformity for values, as well as directions of displacement. Obtained values are consistent with an accuracy of less than $0.5 \mathrm{~mm}$.

\subsection{Vertical Displacements}

Apart from longitudinal displacements, vertical displacement values were compared. Obtained results are presented in form of graphs and compiled in Table 1. Similarly to horizontal displacements, high conformity of results can be observed in vertical displacements. Obtained values are consistent with an accuracy of less than $0.5 \mathrm{~mm}$. Points located closer to the crown were lowered, whereas points located at the base of the section were raised, which can confirm influence onto structure by the foundations/sub-base.

It has been noticed that 2D DIC method is a surface method, continuous-not discreet like classical geodetic method. Despite many advantages, the $2 \mathrm{D}$
DIC method lacks unambiguous evaluation of precision and relevance of designated displacements, which is necessary for possibilities of utilization in technical control of large engineered objects.

For better visualization of advantages and drawbacks of researched methods, results of comparison appraisal are presented in Table 2. Each considered aspect received an appropriate mark for each method.

\section{Conclusions}

Measuring experiment described in this paper was carried out on Rożnów Concrete Dam. During work of power station turbo-plants downstream, face of the dam was monitored in attention to the possible displacements in direction parallel to the structure axis. For the measurements, geodetic measurement techniques and 2D DIC method were used, comparing results in relation to credibility, efficiency and precision for such kind of applications (accuracy of calculation level at 1 2 mm). Based on research results, it has been affirmed that both methods are appropriate, and such kind of research can be used as an additional 


\section{Application of Digital Image Correlation and Geodetic Displacement Measuring Methods to Monitor Water Dam Behavior under Dynamic Load}

element of the assessment on how the dynamic load in form of starting flows through turbo-plants affects structural conditions.

Additional comparison of both methods in relation to their applicability and usability in monitoring surveys of water dams was conducted. Above all, it can be stated that geodetic methods are more reliable due to possibility of stable positioning of the instrument. For a digital camera, a special stand in a close proximity from surveyed object should be prepared. Furthermore, geodetic methods allow to establish objects displacement in the 3D space, especially in direction perpendicular to the objects crown axis (along the $x$-axis). Actions in this direction have the greatest influence onto statics and safety of the object. 2D DIC method allows to visualize deformations only in the $2 \mathrm{D}$ space, in plane parallel to the external surface of the structure, however, the method can be extended into $3 \mathrm{D}$ by use of two synchronized cameras set in a stereo setup [7].

Applicability of 2D DIC method in such measurements will be improved by development of a software version designated for mobile devices (such as smart phones, smart cameras or tablets). The mobile version of 2D DIC with built-in procedures for merging of data distributed in time is currently under development. It should be also marked that the tacheometric method is more reliable but is more laborious. Therefore, each one of the presented method has its merits and disadvantages but used together make up a valuable source of information about the object.

\section{Acknowledgments}

The work is partially financed by the statutory funds of Warsaw University of Technology and Method Foundation for Polish Science within IMPULS programme.

\section{References}

[1] Lewandowski, S. 2006. "Hydropower in Poland: Current State and Prospects for Development." Ekopartner 1: 10-6. (in Polish)

[2] Bryś, H. 1996. Geodetic Measurements of Deformation and Displacement of Dams. Warsaw: Scientific Publishing House PWN. (in Polish)

[3] Lang, W. 1929. Deformation Measurements on Dams According to the Methods of Geodesy: On Behalf of the Department of Topography of the Federal Military Department. Berlin: Publishing House of the German Department of Topography. (in Germany)

[4] Sutton, M., Orteu, J. J., and Schreier, H. 2009. Image Correlation for Shape, Motion and Deformation Measurements. Berlin: Springer.

[5] Hartley, R., and Zisserman, A. 2003. Multiple View Geometry in Computer Vision. Cambridge: Cambridge University Press.

[6] Ma, Y., Soatto, S., Kosecká, J., and Sastry, S. An Invitation to $3 D$ Vision: From Images to Geometric Models (Interdisciplinary Applied Mathematics). Berlin: Springer.

[7] Malesa, M., and Kujawinska, M. 2012. "Modified Two-Dimensional Digital Image Correlation Method with Capability of Merging of Data Distributed in Time." Applied Optics 51 (6): 8641-55.

[8] Fischler, M., and Bolles, R. 1981. "Random Sample Consensus: A Paradigm for Model Fitting with Applications to Image Analysis and Automated Cartography." Communications of ACM 24 (6): 281-395

[9] Prószyński, W., and Kwaśniak, M. 2006. Basics of Geodetic Determination Displacements. The Concepts and Elements of the Methodology. Warsaw: Warsaw University of Technology Publishing House. (in Polish) 